\title{
Calculation of symbol error rate in a telecommunication system based on amplitude modulation of many components
}

\author{
Horbatyi I. V. \\ Lviv Polytechnic National University, \\ 12 S. Bandera Str., 79013, Lviv, Ukraine
}

(Received 20 March 2020; Revised 27 January 2021; Accepted 28 January 2021)

\begin{abstract}
The known analytical equations for calculating the symbol error rate (SER) in the M-ary telecommunication system are considered. The analytical equations for calculating SER in a telecommunication system based on M-ary amplitude modulation of many components (M-AMMC) and other varieties of amplitude-phase shift keying with arbitrary number and arbitrary location of signal points of the signal constellation, as well as under the action of additive white Gaussian noise in a communication line are proposed. According to the results of the research, it is found that the proposed equations allow us to increase the accuracy of calculating SER when using M-AMMC and other varieties of amplitude-phase shift keying compared to known analytical equations.
\end{abstract}

Keywords: M-ary telecommunication system, symbol error rate, amplitude modulation of many components.

2010 MSC: 60G15, 60G35, 93A13, 93A40, 93E10

DOI: $10.23939 / \mathrm{mmc} 2021.02 .137$

\section{Introduction}

Quality is an important indicator of a telecommunication system. It is a set of properties of the telecommunication system that determine the ability to meet certain needs in accordance with the purpose of this system. The main quality characteristics include reliability, safety and certainty. A significant number of publications are devoted to the study of the reliability of telecommunication systems and their components [1-5]. Security issues are discussed in [6]. Also, modern publications pay considerable attention to the certainty of telecommunication systems and their components, in particular to ensure a low error rate in data transmission at the presence of noise in a communication line $[7-10]$.

The error rate at a given data rate is an important characteristic of a data transmission telecommunication system. There are the symbol error rate (SER) and the bit error rate (BER). SER $P_{\text {sym }}$ is calculated as the ratio of the number of symbols transmitted with an error to the total number of transmitted symbols in the data transmission telecommunication system. BER $P_{b}$ is equal to the ratio of the number of data bits transmitted with an error to the total number of transmitted data bits in the data transmission telecommunication system.

To accurately determine SER (BER), it is necessary that the total number of transmitted symbols (bits) go to infinity. Therefore, the use of experimental methods for measuring the error rate is difficult. In this case, the analytical equations for theoretical calculation of error rate are of great interest. They make it possible to predict theoretically the error rate when using certain types of signals and the presence of certain types of noise in a communication line that is a part of a telecommunication system.

It is necessary to ensure a minimum error rate in a data transmission telecommunication system at the presence of noise, at a given data rate, signal power and operating frequency band of a system, which is affected by the characteristics of the components of such a system. In most cases, when building mathematical model of a telecommunication system, it is assumed that there is an additive 
white Gaussian noise (AWGN) in the communication line. The precision of a telecommunication system with such noise is higher when BER $P_{b}$ after signal detection and decoding is lower, and the efficiency of this system is higher when the required signal energy over a time interval equals the transmission time of one data bit to the one-sided noise power spectral density ratio (signal to noise ratio - SNR) $E_{b} / N_{0}$ of AWGN is lower to provide a given BER $P_{b}$. Varieties of signal modulation and corrective coding used in a telecommunication system significantly affect the error rate.

There are a number of known equations for calculating the error rate when applying certain varieties of modulation and corrective coding. The equation for calculation SER at using of M-ary amplitude shift keying (M-ASK) with evenly spaced signal points of the signal constellation on the amplitude axis is given in [11]. The formula for calculating SER when using M-ary phase shift keying (M-PSK) with uniformly distant signal points of the signal constellation on a circle of unit radius on the signal plane is given in [12]. The equation for calculation SER is given in [11] and the equation for calculation BER is given in [12] at using M-ary quadrature amplitude modulation (M-QAM) with uniformly distant signal points of the signal constellation on the signal plane, which fit into a square, with the number of points $2^{k}$, where $k$ is an even integer. However, such formulas do not allow to accurately calculate the error rate at other configurations of signal constellations, at the uneven distant signal points of the signal constellation and the number of points that is not equal to $2^{k}$. However, varieties of signal modulation can be used in modern telecommunication systems, which have signal constellations that are different from the classic known constellations. As an example of the use of a new variety of amplitude-phase modulation, the amplitude modulation of many components (AMMC) can serve [13]. In this case, the question of predicting the error rate at using of such varieties of modulation in a telecommunication system arises.

The aim of this work is to develop equations that will allow to theoretically calculate the error rate in a telecommunication system based on M-ary AMMC (M-AMMC) and other varieties of amplitude-phase shift keying with arbitrary number and arbitrary location of signal points of the signal constellation on the signal plane.

\section{Error rate in the binary telecommunication system}

Ensuring a minimum error rate at a given data rate and operating frequency band is important in the data transmission process, which is influenced by the characteristics of the components of a telecommunication system, and especially the detector $[11,12,14]$. The task of the detector is to recognize the received signal with the minimum number of errors. The detection process will be affected by noise in the communication line, which causes errors. Consider the equation for calculating SER in a binary telecommunication system. Assume that a binary signal of the next form is transmitted in such a system during the time interval $\left(0, T_{\text {sym }}\right)$ :

$$
u_{s}(t)= \begin{cases}U_{s 1}, & 0<t \leqslant T_{\text {sym }} \text { for " } 1 " \\ U_{s 2}, & 0<t \leqslant T_{\text {sym }} \text { for " } 0 "\end{cases}
$$

that is, we can say that one of the two possible symbols $U_{s 1}$ and $U_{s 2}$ or one data bit are transmitted during the time interval $\left(0, T_{\text {sym }}\right)$ in this system. In this case, the transmission time of one symbol $T_{\text {sym }}$ is equal to the transmission time of one data bit $T_{b}\left(T_{\text {sym }}=T_{b}\right)$.

In addition to the signal $u_{s}(t)$, there will also be noise in the real communication line. If AWGN $u_{n}(t)$ with standard deviation $\sigma_{0}$ is present in the communication line, then the density of the conditional probability of signal transmission in a telecommunication system with additive noise $u_{s n}(t)$, provided that the signal $u_{s}(t)=U_{s 1}$ was transmitted, is equal to:

$$
p\left(s_{s n}(t) \mid U_{s 1}\right)=\frac{1}{\sigma_{0} \sqrt{2 \pi}} \exp \left[-\frac{\left(u_{s n}(t)-U_{s 1}\right)^{2}}{2 \sigma_{0}^{2}}\right],
$$

Mathematical Modeling and Computing, Vol. 8, No. 2, pp. 137-149 (2021) 
and for the signal $u_{s}(t)=U_{s 2}$, respectively, is:

$$
p\left(s_{s n}(t) \mid U_{s 2}\right)=\frac{1}{\sigma_{0} \sqrt{2 \pi}} \exp \left[-\frac{\left(u_{s n}(t)-U_{s 2}\right)^{2}}{2 \sigma_{0}^{2}}\right] .
$$

At the detection stage, a hypothesis $H_{1}$ is selected if $u_{s n}(t)>\gamma_{t}$ or a hypothesis $H_{2}$ if $u_{s n}(t)<\gamma_{t}$ by comparing the signal $u_{s n}(t)$ at the input of the decision device with the threshold value $\gamma_{t}$ of such a device. It is necessary to minimize the error rate when choosing a threshold value $\gamma_{t}$ for decision-making when detecting a signal.

The process of choosing hypotheses can be expressed through conditional probabilities:

$$
P\left(U_{s_{1}} \mid u_{s n}(t)\right) \underset{H_{2}}{\stackrel{H_{1}}{\gtrless}} P\left(U_{s 2} \mid u_{s n}(t)\right)
$$

where $P\left(U_{s 1} \mid u_{s n}(t)\right)$ is the conditional probability of transmission of the symbol $U_{s 1}$, provided that the signal $u_{s n}(t)$ was transmitted; $P\left(U_{s 2} \mid u_{s n}(t)\right)$ is the conditional probability of transmission of the symbol $U_{s 2}$, provided that the signal $u_{s n}(t)$ was transmitted.

This equation states that it is necessary to choose a hypothesis $H_{1}$ if the conditional probability $P\left(U_{s 1} \mid u_{s n}(t)\right)$ is greater than the conditional probability $P\left(U_{s 2} \mid u_{s n}(t)\right)$, and the hypothesis $H_{2}$ otherwise.

Using the Bayesian formula $[15,16]$, equation (4) can be represented as

$$
\frac{P\left(U_{s 1}\right) p\left(u_{s n}(t) \mid U_{s 1}\right)}{P\left(u_{s n}(t)\right)} \underset{H_{2}}{\stackrel{H_{1}}{\gtrless}} \frac{P\left(U_{s 2}\right) p\left(u_{s n}(t) \mid U_{s 2}\right)}{P\left(u_{s n}(t)\right)},
$$

where $P\left(U_{s 1}\right)$ is the probability that the symbol $U_{s 1}$ was transmitted, $P\left(U_{s 2}\right)$ is the probability that the symbol $U_{s 2}$ was transmitted, $P\left(u_{s n}(t)\right)$ is the probability that the signal $u_{s n}(t)$ was transmitted.

Formula (4) can be represented by the ratio of plausibility functions $p\left(u_{s n}(t) \mid U_{s 1}\right)$ and $p\left(u_{s n}(t) \mid U_{s 2}\right)$ :

$$
\frac{p\left(u_{s n}(t) \mid U_{s 1}\right)}{p\left(u_{s n}(t) \mid U_{s 2}\right)} \underset{H_{2}}{\stackrel{H_{1}}{\gtrless}} \frac{P\left(U_{s 2}\right)}{P\left(U_{s 1}\right)} .
$$

We can obtain the following formula from (5) provided under equal probabilities $P\left(U_{s 1}\right)=P\left(U_{s 2}\right)$ taking into account (2) and (3):

$$
u_{s n}(t) \stackrel{H_{1}}{\underset{H_{2}}{\gtrless}} \frac{U_{s 1}+U_{s 2}}{2}=\gamma_{o p t},
$$

where $\gamma_{\text {opt }}$ is the optimal threshold value.

Thus, $\gamma_{o p t}$ is the optimal threshold value according to the criterion of the minimum error rate.

SER when receiving a binary signal using the total probability formula [15] is equal to:

$$
P_{\text {sym }}=P\left(U_{s 1}\right) P\left(\varepsilon \mid U_{s 1}\right)+P\left(U_{s 2}\right) P\left(\varepsilon \mid U_{s 2}\right),
$$

where $P\left(\varepsilon \mid U_{s 1}\right)$ is the conditional probability of an error $\varepsilon$ when transmitting the symbol $U_{s 1}, P\left(\varepsilon \mid U_{s 2}\right)$ is the conditional probability of an error $\varepsilon$ when transmitting the symbol $U_{s 2}$.

Thus, an error $\varepsilon$ will occur when transmitting a symbol $U_{s 1}$ if a signal at the input of the deciding device $u_{s n}(t)>\gamma_{o p t}$ due to noise in the communication line, and when transmitting a symbol $U_{s 2}$ if a signal at the input of the deciding device $u_{s n}(t)<\gamma_{\text {opt }}$ due to noise in the communication line. The conditional probability of error $\varepsilon$ in the first case is equal to:

$$
P\left(\varepsilon \mid U_{s 1}\right)=\int_{-\infty}^{\gamma_{o p t}} p\left(u_{s n}(t) \mid U_{s 1}\right) d u_{s n}(t),
$$


and in the second case similarly:

$$
P\left(\varepsilon \mid U_{s 2}\right)=\int_{\gamma_{o p t}}^{\infty} p\left(u_{s n}(t) \mid U_{s 2}\right) d u_{s n}(t) .
$$

Assuming that the probability densities $p\left(u_{s n}(t) \mid U_{s 1}\right)$ and $p\left(u_{s n}(t) \mid U_{s 2}\right)$ are symmetric and $P\left(U_{s 1}\right)=P\left(U_{s 2}\right)=1 / 2$, we obtain:

$$
P_{\text {sym }}=\frac{1}{2} P\left(\varepsilon \mid U_{s 1}\right)+\frac{1}{2} P\left(\varepsilon \mid U_{s 2}\right)=P\left(\varepsilon \mid U_{s 1}\right)=P\left(\varepsilon \mid U_{s 2}\right) .
$$

Taking into account (6) and (9), we obtain:

$$
P_{\text {sym }}=\int_{\gamma_{o p t}=\left(U_{s 1}+U_{s 2}\right) / 2}^{\infty} p\left(u_{s n}(t) \mid U_{s 2}\right) d u_{s n}(t) .
$$

We obtain for a system with AWGN, taking into account (3):

$$
P_{\text {sym }}=\int_{\gamma_{\text {opt }}=\left(U_{s 1}+U_{s 2}\right) / 2}^{\infty} \frac{1}{\sigma_{0} \sqrt{2 \pi}} \exp \left\{-\frac{\left(u_{s n}(t)-U_{s 2}\right)^{2}}{2 \sigma_{0}^{2}}\right\} d u_{s n}(t) .
$$

Let us make a replacement $u=\left(u_{s n}(t)-U_{s 2}\right) / \sigma_{0}$. Then $\sigma_{0} d u=d u_{s n}(t)$ and we obtain the following equation to calculate SER after replacing the variable in (12):

$$
P_{s y m}=\frac{1}{\sqrt{2 \pi}} \int_{\left(U_{s 1}-U_{s 2}\right) / 2 \sigma_{0}}^{\infty} \exp \left\{-\frac{u^{2}}{2}\right\} d u=Q_{1}\left(\frac{U_{s 1}-U_{s 2}}{2 \sigma_{0}}\right)
$$

where $Q_{1}(x)$ is the function determined according to [12] as

$$
Q_{1}(x)=\frac{1}{\sqrt{2 \pi}} \int_{x}^{\infty} \exp \left\{-\frac{u^{2}}{2}\right\} d u
$$

The dispersion of AWGN with the one-sided power spectral density $N_{0}$ passing through the multiplier or filter is equal $\sigma_{0}^{2}=N_{0} / 2$ according to [12], so equation (13) can be represented as

$$
P_{\text {sym }}=Q_{1}\left(\sqrt{\frac{d^{2}}{2 N_{0}}}\right)
$$

where $d=U_{s 1}-U_{s 2}$ is the distance between two symbols.

Thus, equation (15) makes it possible to determine SER in the binary telecommunication system in the presence of AWGN in the communication line. SER is lowest in the case when the threshold value is optimal and increases when the threshold value deviates from the optimal one. As can be seen from (15), SER in such a system at the optimal threshold level depends on the distance $d$ between the two symbols and the one-sided power spectral density $N_{0}$ of AWGN. It should be noted that SER is equal to BER in such system.

\section{Error rate when using widely used varieties of amplitude shift keying, phase shift keying and amplitude-phase shift keying}

Consider the equation for calculating SER in a telecommunication system based on widely used varieties of amplitude shift keying, phase shift keying and amplitude-phase shift keying. 
Let us investigate SER in a telecommunication system with AWGN in a communication line when using binary amplitude shift keying (BASK). A unipolar BASK signal can have one of two possible values of amplitude $U_{1}=0$ or $U_{2}=\sqrt{E_{b}}$ ( $E_{b}$ is the signal energy at a time interval equal to the transmission time of one data bit), with the distance between the symbols $d=U_{1}-U_{2}=\sqrt{E_{b}}$. In such a system, one data bit is transmitted during the transmission time of one symbol $T_{s y m}$, i.e. $T_{s y m}=T_{b}$.

SER and BER in the telecommunication system when using BASK in accordance with (15) is

$$
P_{\text {sym }_{B A S K}}=P_{b_{B A S K}}=Q_{1}\left(\sqrt{\frac{E_{b}}{2 N_{0}}}\right) .
$$

The $\log _{2}(M)$ data bits are transmitted during the transmission time of one symbol $T_{\text {sym }}$ in a telecommunication system when using M-ary amplitude shift keying (M-ASK) with $M$ possible evenly spaced amplitude levels of signal (maximum amplitude value is $U_{\max }=\sqrt{E_{b}}$, minimum amplitude value is $\left.U_{\min }=-\sqrt{E_{b}}\right)$, and SER in such a system is equal to [11]

$$
P_{\text {sym }_{A S K}}=\frac{2(M-1)}{M} Q_{1}\left(\sqrt{\frac{6 \log _{2}(M) E_{b_{a v r}}}{\left(M^{2}-1\right) N_{0}}}\right),
$$

where $E_{b_{a v r}}=\frac{1}{M K} \sum_{m=1}^{M} \sum_{k=1}^{K} E_{b_{m k}}$ is the average signal energy at a time interval equal to the transmission time of one data bit, $K=\log _{2}(M)$ is the number of data bits that one of $M$ possible symbols carries in the M-ary telecommunication system, $E_{b_{m k}}$ is the signal energy at a time interval equal to the transmission time of $k$ th data bit when transmitting $m$ th symbol.

In the case where $M$ symbols are used for transmission, and the symbols are matched with a certain bit sequence formed using the Gray code, the relationship between the BER and SER at $P_{\text {sym }} \ll 1$ will be as follows [12]:

$$
P_{b}=\frac{P_{\text {sym }}}{\log _{2}(M)} .
$$

Let us investigate SER in a telecommunication system with AWGN in a communication line when using binary phase shift keying (BPSK) [11,12]. Let one of the two possible symbols $U_{s 1}$ and $U_{s 2}$ or one data bit be transmitted in a telecommunication system during the time interval $\left(0, T_{\text {sym }}\right) . T_{\text {sym }}=T_{b}$ in this case. Antipodal signals with the same amplitudes $U_{1}=U_{2}=\sqrt{E_{b}}$ and phases $\varphi_{1}=0, \varphi_{2}=\pi$ are used when using such modulation. The distance between the symbols is equal to $d=2 U_{0}=2 \sqrt{E_{b}}$, because antipodal signals are used at the implementation of BPSK. In this case

$$
P_{s y m_{B P S K}}=P_{b_{B P S K}}=Q_{1}\left(\sqrt{\frac{2 E_{b}}{N_{0}}}\right) .
$$

SER when using M-ary phase shift keying (M-PSK) with $M$ possible evenly spaced initial phases of the signals at $M>2$ is [12]:

$$
P_{\text {sym }_{P S K}} \approx 2 Q_{1}\left(\sqrt{\frac{2 E_{\text {sym }}}{N_{0}}} \sin \left(\frac{\pi}{M}\right)\right)=2 Q_{1}\left(\sqrt{\frac{2 \log _{2}(M) E_{b}}{N_{0}}} \sin \left(\frac{\pi}{M}\right)\right),
$$

where $E_{\text {sym }}$ is the signal energy at a time interval equal to the transmission time of one symbol.

Quadrature amplitude modulation (QAM) is a variety of amplitude-phase shift keying. SER in a telecommunication system when using M-ary quadrature amplitude modulation (M-QAM) with $M$ possible symbols ( $M=2^{k}$ where $k$ is an even integer) and evenly spaced amplitude levels of modulating 
signals on both channels I and Q of the modulator is equal to [11]

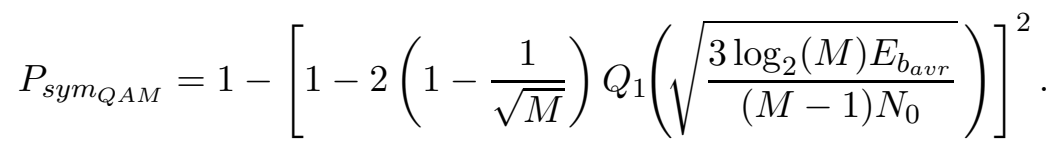

BER when using M-QAM with $M$ possible symbols $\left(M=2^{k}\right.$ where $k$ is an even integer) can be calculated using another known equation [12]:

$$
P_{b_{Q A M}}=\frac{2\left(1-\frac{1}{\sqrt{M}}\right)}{\log _{2}(M)} Q_{1}\left(\sqrt{\frac{6 \log _{2}(\sqrt{M})}{M-1} \frac{E_{b_{a x r}}}{N_{0}}}\right) .
$$

\section{Error rate when using amplitude modulation of many components}

Consider the equation for calculating SER in a telecommunication system based on M-AMMC. This new proposed modulation is a variety of amplitude-phase shift keying and is characterized by high efficiency and, as a rule, signal constellations of non-rectangular form [13]. An example of a signal constellation of the 16-ary amplitude modulation of many components signal with three components having the shift of amplitude levels of modulating signals (16-AMMC signal with three components having the shift of amplitude levels of modulating signals) is shown in Fig. 1.

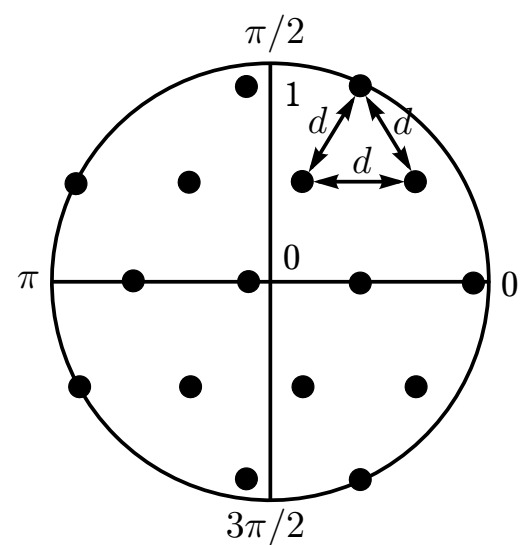

Fig. 1. Signal constellation of 16AMMC signal with three components having the shift of amplitude levels of modulating signals.

The signal points of the signal constellation of the 16-AMMC signal with three components having the shift of amplitude levels of modulating signals fit into a hexagon (see Fig. 1), in contrast to the signal constellation of the 16-ary quadrature amplitude modulation (16-QAM) signal, the signal points of which fit into a square [13].

$\mathrm{SER}$ in a telecommunication system based on M-AMMC for the

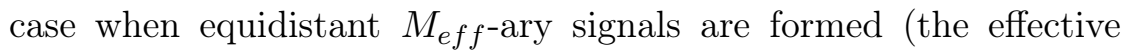
number of symbols $M_{\text {eff }}$ corresponding to unique signal points of the signal constellation are used) taking into account $[11,17]$ does not exceed the value that can be calculated using the following equation:

$$
P_{s y m_{A M M C}} \leqslant 6 \frac{M_{e f f}-1}{M_{e f f}} Q_{1}\left(\sqrt{\frac{K_{E} \log _{2}\left(M_{e f f}\right)}{2} \frac{E_{b_{a v r}}}{N_{0}}}\right),
$$

where $K_{E}$ is the coefficient equal to the ratio of the minimally possible energy of the difference signal $\Delta E_{\text {min }}$ for two symbols corresponding to adjacent points of the signal constellation to the average energy $E_{\text {symavr }}=\frac{1}{M_{e f f}} \sum_{i=1}^{M_{e f f}} E_{s y m_{i}}$ of all $i$ th signals with energies $E_{\text {sym }}$ at a time intervals equal to the transmission time of the $i$ th symbols for $M_{e f f}$ symbols corresponding to unique signal points of the signal constellation.

It is expedient to use such the upper limit $[11,17]$ of the error rate of arbitrary $M_{e f f}$-ary signals to calculate a more accurate value of SER when using M-AMMC:

$$
P_{\text {sym }_{M}} \leqslant\left(M_{e f f}-1\right) Q_{1}\left(\sqrt{\frac{\left(d_{\mathrm{min}}\right)^{2}}{2 N_{0}}}\right)=\left(M_{e f f}-1\right) Q_{1}\left(\sqrt{\frac{\Delta E_{\mathrm{min}}}{2 N_{0}}}\right),
$$

where $d_{\text {min }}$ is the minimally possible Euclidean distance between adjacent points of the signal constellation, $\Delta E_{\min }$ is the minimally possible energy of the difference signal for two symbols corresponding to adjacent points of the signal constellation. 
The energy of the difference signal for two arbitrary $i$ th and $j$ th symbols is equal to

$$
\begin{aligned}
\Delta E_{i j} & =\int_{0}^{T}\left(s_{i}(t)-s_{j}(t)\right)^{2} d t=\int_{0}^{T}\left(s_{i}(t)\right)^{2} d t-2 \int_{0}^{T} s_{i}(t) s_{j}(t) d t+\int_{0}^{T}\left(s_{j}(t)\right)^{2} d t \\
& =E_{\text {sym }_{i}}-2 E_{\text {sym }_{i j}}+E_{\text {sym }_{j}},
\end{aligned}
$$

where $s_{i}(t)$ and $s_{j}(t)$ are the signals corresponding to $i$ th and $j$ th symbols, $T$ is the transmission time of the signals $s_{i}(t)$ or $s_{j}(t)$ that is equal to the transmission time of one symbol $T_{s y m}, E_{s y m_{i}}$ and $E_{s y m_{j}}$ are the signal energies corresponding to $i$ th and $j$ th symbols, $E_{s y m_{i j}}$ is the similarity coefficient of the signals corresponding to $i$ th and $j$ th symbols.

Let us represent SER when detecting arbitrary $M_{e f f}$-ary signals (24) as a function of $E_{b_{a v r}} / N_{0}$. It is seen according to (25) that the minimally possible energy of the difference signal for two symbols in general can be represented in terms of the average signal energy per symbol $E_{\text {symavr }}$ for all $M_{\text {eff }}$ symbols corresponding to a given signal constellation:

$$
\Delta E_{\min }=E_{\text {symavr }} K_{E},
$$

where $K_{E}$ is the proportionality coefficient.

We see from equation (26) that the coefficient $K_{E}$ is equal to the ratio of the minimally possible energy of the difference signal $\Delta E_{\text {min }}$ for two symbols corresponding to adjacent points of the signal constellation to the average signal energy $E_{\text {symavr }}$ for all $M_{\text {eff }}$ symbols corresponding to unique signal points:

$$
K_{E}=\frac{\Delta E_{\min }}{E_{\text {sym }} \text { avr }}=\Delta E_{\min } /\left(\frac{1}{M_{\text {eff }}} \sum_{i=1}^{M_{\text {eff }}} E_{\text {sym }}\right)=\Delta E_{\min } M_{\text {eff }} / \sum_{i=1}^{M_{\text {eff }}} E_{\text {sym }},
$$

where $E_{\text {sym }}$ is the signal energy at a time interval equal to the transmission time of $i$ th symbol.

We will use for further transformations the known relationship [11] between the average signal energy at a time interval equal to the transmission time of one symbol $E_{\text {sym }}$ avr and the average signal energy at a time interval equal to the transmission time of one data bit $E_{b_{a v r}}$ at the effective number $M_{\text {eff }}$ of symbols in the alphabet of the manipulated signal (effective number of points of the signal constellation)

$$
E_{\text {symavr }}=\log _{2}\left(M_{e f f}\right) E_{b_{a v r}}
$$

Using (28), we can show that

$$
P_{\text {sym }} \leqslant\left(M_{e f f}-1\right) Q_{1}\left(\sqrt{\frac{\Delta E_{\min } \log _{2}\left(M_{e f f}\right)}{E_{\text {symavr }}} \frac{E_{b_{a v r}}}{2 N_{0}}}\right) .
$$

Taking into account (27), we obtain

$$
P_{\text {sym }} \leqslant\left(M_{e f f}-1\right) Q_{1}\left(\sqrt{K_{E} \log _{2}\left(M_{e f f}\right) \frac{E_{b_{a v r}}}{2 N_{0}}}\right) .
$$

According to the results of the research, the author found that it is advisable to use the coefficient $n_{a}$ equal to the average number of adjacent points that are located around one of the point of the signal constellation instead of a multiplier $\left(M_{e f f}-1\right)$ to increase the accuracy of calculations. In this case, the author proposes to apply the following equation to calculate SER in a telecommunication system based on M-AMMC when using signals that correspond to a signal constellation with equally 
distant adjacent points [13]:

$$
P_{\text {sym } A M M C} \approx n_{a} Q_{1}\left(\sqrt{K_{E} \log _{2}\left(M_{e f f}\right) \frac{E_{b_{a v r}}}{2 N_{0}}}\right) .
$$

The adequacy of (31) is confirmed by the coincidence of the results of calculating SER when using it for M-QAM and the results obtained using a known formula (21) to calculate SER when using MQAM. Thus, the proposed equation (31) is suitable for calculating SER in a telecommunication system based on M-QAM and M-AMMC at $P_{\text {sym }}{ }_{A M M C}<0.1$.

\section{Error rate when using varieties of amplitude-phase shift keying with arbitrary number and arbitrary location of signal points of the signal constellation}

Consider the proposed equation for calculating SER in a telecommunication system based on varieties of amplitude-phase shift keying at arbitrary number and arbitrary location of signal points of the signal constellation, as well as under the action of AWGN in the communication line, which allows to increase the accuracy in calculating the error rate when using M-AMMC and other varieties of amplitude-phase shift keying.

A number of formulas are given in [17], which allow to calculate SER in the M-ary telecommunication system in which arbitrary $M$ signals are used. Among them there is the formula for calculating the upper limit when using arbitrary $M$ signals, which is called the additive limit:

$$
P_{\text {sym }} \leqslant \sum_{\substack{i=1 \\ i \neq j}}^{M} P_{\text {sym }},
$$

where $P_{\text {sym }}$ is the probability of erroneous receiving during transmission of signal $s_{i}(t), P_{\text {sym }}$ ij is the probability of error in the binary telecommunication system if a signal $s_{j}(t)$ corresponding to the $j$ th symbol $U_{s_{j}}$ was received during the transmission of the signal $s_{i}(t)$ corresponding to the $i$ th symbol $U_{s_{i}}$.

This limit can be simplified by reducing the accuracy:

$$
P_{\text {sym }_{M}} \leqslant(M-1) \max _{(i, j)} P_{s y m_{i j}},
$$

where $P_{s y m_{M}}$ is SER in the M-ary telecommunication system when transmitting any signal, $\max _{(i, j)} P_{s y m_{i j}}$ is the maximum probability of error in all pairs $(i, j)$ in the binary telecommunication system with signals $s_{i}(t)$ and $s_{j}(t)$ corresponding to the $i$ th symbol $U_{s_{i}}$ and $j$ th symbol $U_{s_{j}}$.

In particular, it follows from (33) that for a telecommunication system with AWGN in a communication line during coherent receiving

$$
P_{\text {sym }} \leqslant(M-1) \max _{(i, j)} Q_{1}\left(\sqrt{\frac{\Delta E_{i, j}}{2 N_{0}}}\right),
$$

where $\Delta E_{i, j}$ is the energy of the difference signal for $i$ th symbol $U_{s_{i}}$ and $j$ th symbol $U_{s_{j}}$.

It is easy to see that (34) is equivalent to (24). However, formula (34) does not allow to accurately calculate SER in the M-ary telecommunication system.

We will use the formula of total probability [15] to accurately solve the problem of calculating SER in the M-ary telecommunication system, according to which for such system

$$
P_{\text {sym }_{M}}=\sum_{i=1}^{M} P\left(U_{s_{i}}\right) P\left(\varepsilon \mid U_{s_{i}}\right),
$$


where $P\left(U_{s_{i}}\right)$ is the probability that the symbol $U_{s_{i}}$ is transmitted, $P\left(\varepsilon \mid U_{s_{i}}\right)$ is the conditional probability of error $\varepsilon$ when transmitting the symbol $U_{s_{i}}$.

It is known that the sum of the probability of error and the probability of absence of error (correct receiving) is equal to one. The probability of the absence of an error in the telecommunication system is equal to the multiplication of probabilities of the absence of error $\left(1-P_{s y m, i j}\right)$ for all pairs $(i, j)$ due to the possible perception of the signal $s_{j}(t)$ corresponding to the $j$ th symbol $U_{s_{j}}$ instead of the signal $s_{i}(t)$ corresponding to $i$ th symbol $U_{s_{i}}$ according to the formula for calculating the probability of independent events [16]. Also $\left(1-P_{\text {sym,ii }}\right)=1$, then the conditional probability of an error $\varepsilon$ when transmitting the symbol $U_{s_{i}}$

$$
P\left(\varepsilon \mid U_{s_{i}}\right)=1-\prod_{\substack{i=1 \\ i \neq j}}^{M}\left(1-P_{\text {sym }, i j}\right)
$$

where $P_{s y m, i j}$ is the probability of error in the binary telecommunication system due to the perception of the signal $s_{j}(t)$ corresponding to the $j$ th symbol $U_{s_{j}}$ instead of the signal $s_{i}(t)$ corresponding to the $i$ th symbol $U_{s_{i}}$.

In this case, SER in the M-ary telecommunication system is equal

$$
P_{\text {sym }_{M}}=\sum_{i=1}^{M} P\left(U_{s_{i}}\right)\left(1-\prod_{\substack{i=1 \\ i \neq j}}^{M}\left(1-P_{s y m, i j}\right)\right) .
$$

Therefore, the obtained equation (37) is suitable for calculating SER in the M-ary telecommunication system. In the partial case, SER for the M-ary telecommunication system based on amplitudephase shift keying under the action of AWGN in the communication line, taking into account (31), is equal to

$$
P_{\text {sym }_{M}}=\sum_{i=1}^{M} P\left(U_{s_{i}}\right)\left(1-\prod_{\substack{i=1 \\ i \neq j}}^{M}\left(1-Q_{1}\left(\sqrt{\frac{\Delta E_{i j}}{E_{\text {symavr }}} \log _{2}(M) \frac{E_{b_{a v r}}}{2 N_{0}}}\right)\right)\right) .
$$

Equation (38) can be simplified if all symbols are transmitted with the same probability, i.e. $P\left(U_{s_{i}}\right)=1 / M$ :

$$
P_{\text {sym }_{M}}=\frac{1}{M} \sum_{i=1}^{M}\left(1-\prod_{\substack{i=1 \\ i \neq j}}^{M}\left(1-Q_{1}\left(\sqrt{\frac{\Delta E_{i j}}{E_{\text {symavr }}} \log _{2}(M) \frac{E_{b_{\text {avr }}}}{2 N_{0}}}\right)\right)\right) .
$$

The following limit values of SER (39) are obtained taking into account the limit values for the function (14) $\lim _{x \rightarrow 0} Q_{1}(x)=0.5$ and $\lim _{x \rightarrow \infty} Q_{1}(x)=0$ :

$$
\lim _{\left(E_{b_{a v r}} / N_{0}\right) \rightarrow 0} P_{s y m_{M}}=1-0.5^{M-1}, \quad \lim _{\left(E_{b_{a v r}} / N_{0}\right) \rightarrow \infty} P_{s y m_{M}}=0 .
$$

Thus, the obtained equation (39) is suitable for calculating SER in a telecommunication system with AWGN in a communication line during coherent receiving if M-AMMC with an effective number of symbols $M_{\text {eff }}=M$ or other variety of amplitude-phase shift keying is used in this system.

The lower limit of SER in the M-ary telecommunication system with AWGN in a communication line during coherent receiving was also obtained by simplifying equation (39). This is achieved by calculating the average value of the probability of error due to the possible perception of the signal 
$s_{j}(t)$ corresponding to the $j$ th symbol $U_{s_{j}}$ instead of the signal $s_{i}(t)$ corresponding to the $i$ th symbol $U_{s_{i}}$ :

$$
P_{\text {sym }_{M}}=\frac{1}{M} \sum_{i=1}^{M}\left(\frac{2}{M} \sum_{\substack{i=1 \\ i \neq j}}^{M} Q_{1}\left(\sqrt{\frac{\Delta E_{i j}}{E_{\text {symavr }}} \log _{2}(M) \frac{E_{b_{a v r}}}{2 N_{0}}}\right)\right) .
$$

The following limit values of SER (41) are obtained taking into account the limit values for the function (14) $\lim _{x \rightarrow 0} Q_{1}(x)=0.5$ and $\lim _{x \rightarrow \infty} Q_{1}(x)=0$ :

$$
\lim _{\left(E_{b_{a v r}} / N_{0}\right) \rightarrow 0} P_{\text {sym }_{M}}=\frac{M-1}{M}, \quad \lim _{\left(E_{b_{a v r}} / N_{0}\right) \rightarrow \infty} P_{\text {sym }_{M}}=0 .
$$

Using the obtained equations (39) and (41), a file for calculating SER in a telecommunication system based on AMMC and other varieties of amplitude-phase shift keying with arbitrary number and arbitrary location of signal points of the signal constellation was developed in Mathcad environment. This file provides the forming of a signal constellation with a given number of points and the location of these points on the signal plane for a given set of used signals in analytical form and their corresponding symbols, calculation of average signal energy corresponding to these symbols, calculation of the energy of the difference signal for all combinations of symbol pairs, calculation the probability of erroneous perception of a certain symbol from $M$ possible instead of another symbol with a search of all such possible variants, calculation the values of SER according to (39) and (41) for a given range of signal energy at a time interval equal to the transmission time of one bit to the one-sided AWGN power spectral density ratio.

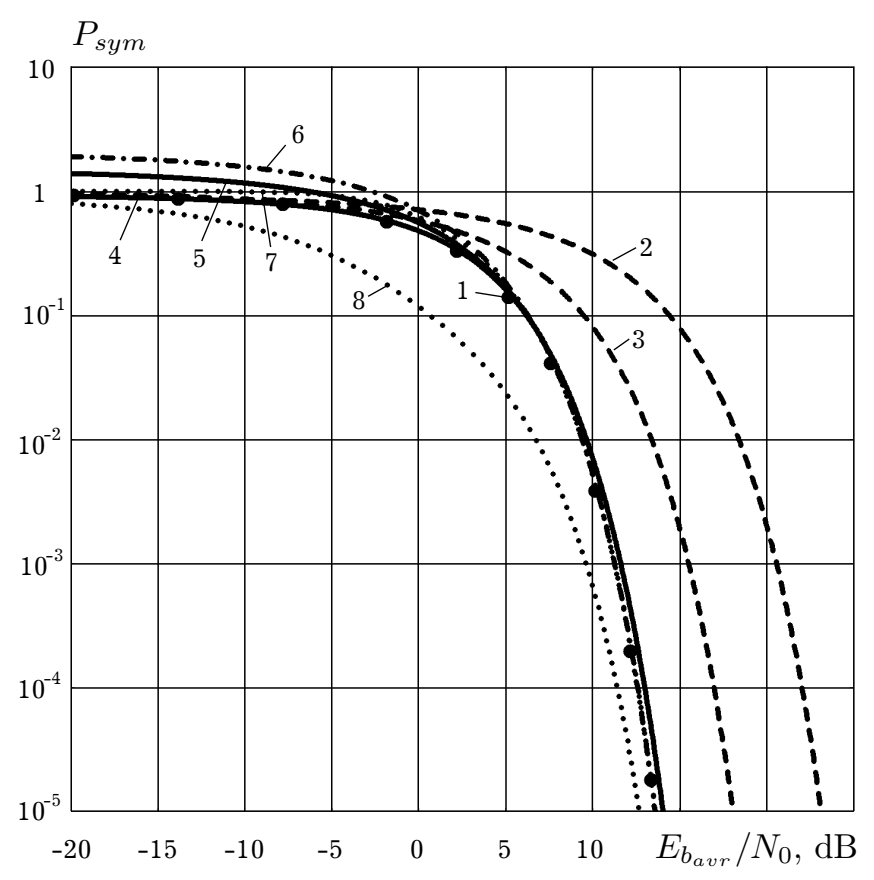

Fig. 2. Dependence of SER $P_{\text {sym }}$ on the ratio $E_{b_{a v r}} / N_{0}$ in the M-ary telecommunication system based on 16AMMC with three components having the shift of amplitude levels of modulating signals when using: 1 , simulation (marked with large dots); 2, equation (17); 3, equation (20); 4 , equation (21); 5 , equation (22) taking into account (18); 6 , equation (31); 7 , equation (39); 8 , equation (41).
Verification of the results obtained in the Mathcad environment using the above known analytical equations and the new proposed analytical equations for calculating SER is carried out by means of simulation mathematical modeling of M-th telecommunication system based on 16-AMMC with three components having the shift of amplitude levels of modulating signals. During the simulation in the Mathcad environment, a package of symbols was formed with the probability of occurrence of individual $i$ th symbols, which is described by the uniform distribution law. In the transmitting device of the telecommunication system, these symbols are matched by modulated 16-AMMC signals that have been transmitted over a communication line with AWGN. The signals from the output of a communication line were demodulated and detected in the receiving device of a telecommunication system. SER was calculated based on the results of comparing the transmitted symbol packet with the received symbol packet.

The results of using known analytical equations and new proposed analytical equations to calculate the dependence of SER $P_{\text {sym }}$ on the signal energy at a time interval equal to the transmission time of one data bit to one-sided 
noise power spectral density ratio $E_{b_{a v r}} / N_{0}$ in the M-ary telecommunication system based on 16-AMMC with three components having the shift of amplitude levels of modulating signals are compared with simulation results and shown in Fig. 2.

The relative errors of the results of using of the above analytical equations relative to the simulation results are given in Table 1.

Table 1. Relative errors of the results of using of analytical equations relative to the simulation results.

\begin{tabular}{|c|c|c|c|c|c|c|c|c|}
\hline$E_{b_{\text {avr }}} / N_{0}$, & $P_{\text {sym }}$ & \multicolumn{7}{|c|}{ Relative error when using the equation, \% } \\
\cline { 3 - 9 } $\mathrm{dB}$ & $($ simulation $)$ & $(17)$ & $(20)$ & $(21)$ & $(22)$ and $(18)$ & $(31)$ & $(39)$ & $(41)$ \\
\hline-19.861 & 0.92340 & -1.0 & 3.5 & -1.7 & 50.7 & 106.1 & 8.3 & -14.1 \\
\hline-13.838 & 0.87205 & 2.2 & 4.4 & -0.1 & 47.2 & 100.1 & 14.5 & -24.7 \\
\hline-7.848 & 0.78320 & 7.9 & 5.1 & 0.4 & 37.3 & 84.0 & 25.0 & -45.0 \\
\hline-1.858 & 0.56949 & 32.4 & 15.2 & 2.0 & 23.8 & 59.4 & 35.2 & -69.0 \\
\hline 2.165 & 0.32905 & 97.7 & 45.6 & 3.7 & 14.5 & 37.9 & 26.2 & -80.1 \\
\hline 5.160 & 0.13950 & 288.7 & 127.6 & 8.7 & 13.1 & 23.2 & 17.3 & -84.2 \\
\hline 7.564 & 0.04092 & 962.2 & 358.0 & 18.1 & 19.6 & 12.8 & 11.1 & -85.8 \\
\hline 10.152 & $3.83 \cdot 10^{-3}$ & $7.8 \cdot 10^{3}$ & $1.9 \cdot 10^{3}$ & 56.3 & 56.5 & 12.7 & 13.0 & -85.9 \\
\hline 12.150 & $1.97 \cdot 10^{-4}$ & $1.0 \cdot 10^{5}$ & $1.3 \cdot 10^{4}$ & 121.8 & 121.9 & 12.8 & 13.6 & -85.8 \\
\hline 13.356 & $1.8 \cdot 10^{-5}$ & $8.0 \cdot 10^{5}$ & $5.7 \cdot 10^{4}$ & 162.5 & 162.5 & -1.2 & -0.3 & -87.5 \\
\hline
\end{tabular}

As can be seen from the results of the research, equations (17) for M-ASK and (20) for M-PSK are the least suitable for calculating SER in a telecommunication system based on 16-AMMC with three components having the shift of amplitude levels of modulating signals. The better results can be achieved by applying (21), (22) and (18) for M-QAM, but the relative error will be significant and will be in the range from 18.1 up to $162.5 \%$ in the range of SER $P_{\text {sym }}$ from 0.04092 to $1.8 \cdot 10^{-5}$ according to (28) compared to the simulation results. Good results can be achieved using (31), but the disadvantage of this equation is a significant increase in the relative error at $P_{\text {sym }}>0.1$. The best results were achieved by applying the new proposed equation (39), which allows to calculate SER $P_{\text {sym }}$ in the range of interest from 0.04092 to $1.8 \cdot 10^{-5}$ with a relative error in the range from 11.1 to $0.3 \%$ compared to the simulation results, and in the whole range of possible values with a relative error not exceeding 36\%. Equation (41) can only serve to estimate the lower limit of SER for M-ary telecommunication systems.

Based on the analysis of the obtained results, we can say that the known research formulas for calculating SER in the M-ary telecommunication system are limiting estimates [17] or obtained for specific signal constellations $[11,12]$. In this paper, we consider the M-ary telecommunication system based on M-AMMC and other varieties of amplitude-phase shift keying with signal constellations with arbitrary location and arbitrary number of signal points on the example of 16-AMMC with three components having the shift of amplitude levels of modulating signals and obtained the analytical formulas for this case. The Mathcad environment was used to apply these formulas in practice. This is why the accuracy of the proposed analytical formulas is higher for constellations for which there are no exact formulas.

\section{Conclusions}

The analytical equation was proposed for the calculation of SER in the M-ary telecommunication system when transmitting data in such a system with the help of $M$ arbitrary signals.

As a partial case of such equation, the analytical equation for the calculation of SER in the M-ary telecommunication system based on M-AMMC and other varieties of amplitude-phase shift keying was proposed. This equation makes it possible to calculate SER in such a system with AWGN in a communication line during coherent receiving if one of varieties of amplitude-phase shift keying with 
arbitrary number and arbitrary location of signal points of the signal constellation used in this system. According to the research results, it is established that the proposed equation allows increasing the accuracy of calculating SER in the M-ary telecommunication system compared to the known equations. The relative error of the calculation results compared to the simulation results is in the range from 11.1 to $0.3 \%$ in the range of SER from 0.04092 to $1.8 \cdot 10^{-5}$, which is interesting for practice.

The proposed analytical equations are suitable for use at the design stage of high-quality data transmission telecommunication systems.

[1] Bobalo Yu. Ya., Horbatyi I. V., Kiselychnyk M. D., Medynsky I. P., Melen M. V. Semi-Markov reliability model of functioning of wireless telecommunication system with complex control system. Mathematical Modeling and Computing. 6 (2), 192-210 (2019).

[2] Bobalo Y., Nedostup L., Kiselychnyk M., Melen M. Optimization of processes designed for ensuring the quality and reliability of electronics using cumulative models of defectiveness and total production expenses. Przeglad Elektrotechniczny. 92 (1), 155-158 (2016).

[3] Bobalo Y., Seniv M., Symets I. Algorithms of automated formulation of the operability condition of complex technical systems. 2018 XIV-th International Conference on Perspective Technologies and Methods in MEMS Design (MEMSTECH). 14-17 (2018).

[4] Bobalo Y., Seniv M., Yakovyna V., Symets I. Method of Reliability Block Diagram Visualization and Automated Construction of Technical System Operability Condition. Advances in Intelligent Systems and Computing III. 871, 599-610 (2019).

[5] Seniv M., Mykuliak A., Senechko A. Recursive algorithm of traversing reliability block diagram for creation reliability and refuse logical expressions. 2016 XII International Conference on Perspective Technologies and Methods in MEMS Design (MEMSTECH). 199-201 (2016).

[6] Toliupa S., Parkhomenko I., Shvedova H. Security and regulatory aspects of the critical infrastructure objects functioning and cyberpower level assesment. 2019 3rd International Conference on Advanced Information and Communications Technologies (AICT). 463-468 (2019).

[7] Weiss E., Bykhovsky D., Arnon Sh. Symbol error rate model for communication using femtosecond pulses for space applications. IEEE Photonics Technology Letters. 28 (12), 1286-1289 (2016).

[8] Yansheng Y., Hong S., Liufeng D., Guangchun F., Xiaomin L., Xiyan T. Influences of delay unit on bite error rate in the BDPSK phase comparing demodulation. 2011 International Conference on Consumer Electronics, Communications and Networks (CECNet). 5016-5019 (2011).

[9] Saiko V., Toliupa S., Nakonechnyi V., Dakov S. The method for reducing probability of incorrect data reception in radio channels of terahertz frequency range. 2018 14th International Conference on Advanced Trends in Radioelecrtronics, Telecommunications and Computer Engineering (TCSET). 1043-1046 (2018).

[10] Bondariev A., Maksymiv I. Method of improvement of quality indexes of detecting in cellular communication systems. Elektronika ir Elektrotechnika. 18 (10), 85-88 (2012).

[11] Proakis J. Digital Communications. New York, McGraw-Hill (2001).

[12] Sklar B. Digital Communications. Fundamentals and Applications. Upper Sadle River, New Jersey, Prentice Hall (2001).

[13] Gorbatyy I. V. Optimization of signal-code constructions using the maximum efficiency criterion. Radioelectronics and Communications Systems. 56 (12), 560-567 (2013).

[14] Ziuko A. G., Klovskii D. D., Nazarov M. V., Fink L. M. Teoriia peredachi signalov: uchebnik dlia vuzov. Moscow, Radio i sviaz (1986), (in Russian).

[15] Zwillinger D., Kokoska S. CRC Standard Probability and Statistics Tables and Formulae. New York, CRC Press (2000).

[16] Florescu I. Probability and Stochastic Processes. New Jersey, Wiley (2014).

[17] Korzhik V. I., Fink L. M., Shchelkunov K. N. Raschet pomekhoustoichivosti sistem peredachi diskretnykh soobshchenii: spravochnik. Pod red. L. M. Finka. Moscow, Radio i sviaz (1981), (in Russian). 


\title{
Обчислення ймовірності символьної помилки в телекомунікаційній системі на основі амплітудної модуляції багатьох складових
}

\author{
Горбатий I. B. \\ Національний університет "Львівсъка політехніка", \\ вул. С. Бандери, 12, 79013, Лъвів, Україна
}

Розглянуто відомі аналітичні співвідношення для обчислення ймовірності символьної помилки в М-вій телекомунікаційній системі. Запропоновано аналітичні співвідношення для обсчислення ймовірності символьної помилки в телекомунікаційній системі на основі М-позиційної амплітудної модуляції багатьох складових (М-АМБС) та інших різновидів амплітудно-фазової маніпуляції за довільної кількості та довільного розташування сигнальних точок сигнального сузір'я, а також при дії адитивного білого гаусового шуму в лінії зв'язку. За результатами здійснених досліджень виявлено, що запропоновані співвідношення дають змогу підвищити точність при обчисленні ймовірності символьної помилки при застосуванні М-АМБС та інших різновидів амплітудно-фазової маніпуляції порівняно з відомими співвідношеннями.

Ключові слова: $M$-ва телекомунікаційна система, імовірність символьної помилки, амплітудна модуляиія багатьох складових. 